\title{
MASTLA
}

\section{Elastic-wave radiation from spherical sources}

Howard C. Rodean

December 7, 1979 


\title{
Elastic-wave radiation from spherical sources
}

\author{
Howard C. Rodean
}

Manuscript date: December 7, 1979

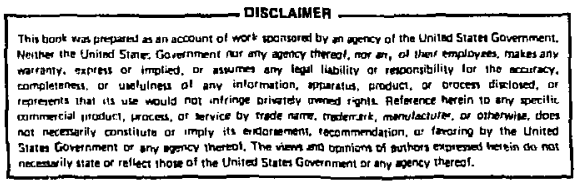

LAWRENCE LIVERMORE LABORATORY University of California - Livermore, Caliliornia - 94550 


\section{CONTENTS}

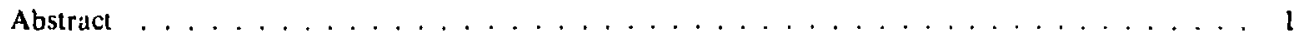

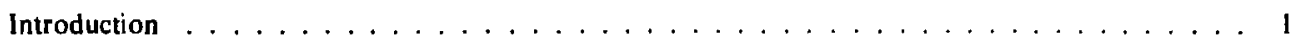

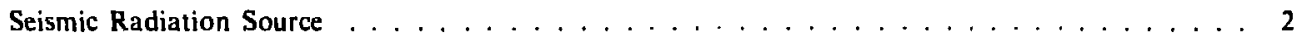

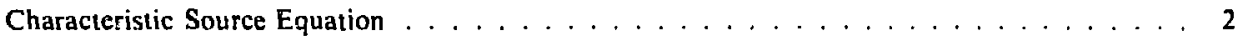

Characteristic Frequencies $\ldots \ldots \ldots \ldots \ldots \ldots$

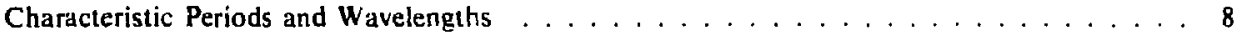

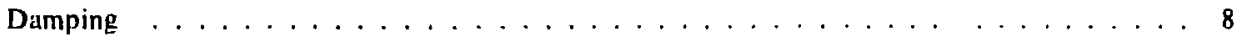

Seismic Radiation Field $\ldots \ldots \ldots \ldots \ldots \ldots \ldots$

Characteristic Field Equations $\ldots \ldots \ldots \ldots \ldots \ldots$

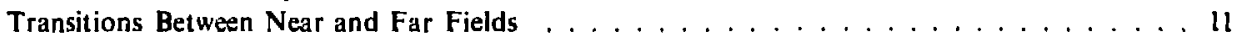

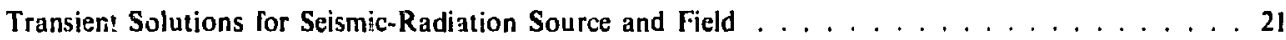

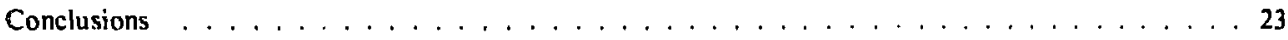

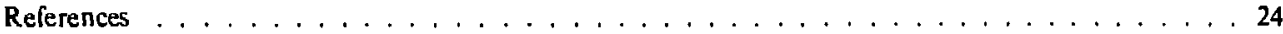




\section{ELASTIC-WAVE RADIATION FROM SPHERICAL SOURCES}

\section{ABSTRACT}

This report treats the radiation of spherical compressional waves from a spherical cavity in an ideal elastic solid. The equations for the radiation source and field are written in terms of the reduced-displacement potential. The source equation is studied in terms of characteristic frequencies, corresponding periods and wavelengths, and damping. The field equations for the stresses, strains, radial displacement, etc., are reviewed with regard to the transitions between the near and far fields, respectively. The natural parameters for defining the dynamic source and field characteristics are $2 b / R$ and $b / a$ in some cases and $a / R$ in others, where $a$ is the compressional-wave velocity, 0 the shear-wave velocity, and $R$ the cavity radius. Transient solutions for stresses, strains, radial displacement, etc., include damped sirusoidal oscillations. The initial- and final-value theorems for the Laplace transform are used to obtain solutions for $\tau$ (the reduced time) $\rightarrow 0+$ (high-frequency, farfield) and $\tau \rightarrow \infty$ (zero-frequency, near-field).

\section{INTRODUCTION}

The infinitesimal point source of dilatation and the finite spherical cavity with variable pressure have been used as seismic source functions in many theoretical studies of seismic waves from earthquakes and explosives. A finite spherical cavity may be replaced by a point source by letting the cavity radius approach zero and the cavity pressure (if nonzero) approach infinity in such a way that the product of the pressure and the cube of the radius remains constant and finite. The subject of this paper is the radiation of compressional waves from a spherical cavity in an infinite homogeneous isotropic elastic solid.

The first mathematical solution of this problem was puolished by Jeffreys (1931), who assumed a step change in cavity pressure for the source function and equal Lame constants $(\lambda=\mu)$ for the elastic solid. The first general solution in terms of $\lambda$ and $\mu$ for a step change in cavity pressure was given by Kawasumi and Yosiyama (1935), who noted the similarity of their solution to that for the damped oscillations of a pendulum. Yoshiyama (1963) (apparently the Yosiyama of the 1935 paper) expanded on this analogy by obtaining a solution in the same form as that of the differential equation for a pendulum with damped oscillations. He wrote his solution in terms of the reduced-displacement potential, although he did not identify his function $[a \cdot f(r)]$ as such, where $\tau$ is the reduced time. Blake (1952) and Selberg (1952) were the first to use the reduceddisplacement potential in obtaining solutions for spherical-wave propagation, but neither used that term for their respective functions $[A \cdot f(\tau)]$ and $\left[c^{2} \psi(\tau)\right]$. Herbst, Werth, and Springer (1961) were the first to apply the term "reduced-displacement potential" to the potential function that is a solution of the spherical-wave equation and is not a function of space but of the reduced time alone.

Using the reduced-displacement potential results in the following relation between the rudial stress $\sigma_{\mathrm{r}}$ (the driving function), and the reduced-displacement potential $\mathbf{X}$ (the response function):

$$
\frac{\sigma_{r}(r, \tau)}{4 \mu}=\frac{1}{r^{3}}\left[\left(\frac{r}{2 b}\right)^{2} \frac{\partial^{2} X(\tau)}{\partial \tau^{2}}+\left(\frac{r}{a}\right) \frac{\partial X(\tau)}{\partial \tau}+X(\tau)\right],
$$

where the reduced time 


$$
T=t-(r-R) / a
$$

and

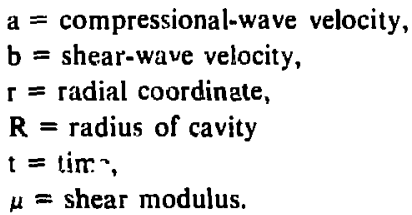

Selberg and Yoshiyama derived equivalent forms of Eq. (1).

\section{SEISMIC RADIATION SOURCE}

The source of spherical compressional waves is defined by Eq. (1) with the substitutions $r=R$ and $\sigma_{r}(\tau)=-P(\tau)$, where $P$ is the cavity pressure. The Laplace transform (Gardner and Barnes, 1942, pp. 127-133) may be used to converi Eq. (1) with these substitutions into a transform equation in which $\hat{\mathrm{P}}(\mathrm{s})$ is the driving transform and $\hat{\mathbf{X}}(\mathrm{s})$ is the response transform:

$$
-\left(\frac{4 \mu}{R^{3}}\right) \frac{\hat{\mathbf{X}}(s)}{\hat{\mathbf{P}}(s)}=\left[\left(\frac{R}{2 b}\right)^{2} s^{2}+\left(\frac{R}{a}\right) s+1\right]^{-1} .
$$

\section{CHARACTERISTIC SOURCE EQUATION}

The polynomial function of the complex variable $s$ on the right side of Eq. (3) is the characteristic function, and the characteristic equation for the source is formed by setting the characteristic function equal to zero. The roots of the characteristic equation in the complex s plane are called the characteristic values:

$$
s=(2 b / R)\left\{-(b / a) \pm i\left[1-(b / a)^{2}\right]^{1 / 2}\right\}
$$

where

$(2 b / R)(b / a)=$ the damping constant,

$(2 b / R)\left[1-(b / a)^{2}\right]^{1 / 2}=$ the characteristic angular frequency with damping, and

$(2 b / R)=$ the characteristic undamped angular frequency (Gardner and Barnes, p. 171).

The word natural is ofien used instead of the word angular in defining the above two frequencies; we will use the word natural in this report. Note that the real and imaginary components of Eq. (4) may be written in terms of only two parameters: one is $2 b / R$, the undamped natural frequency, and the other is

$\mathbf{b} / \mathbf{a}=$ damping ratio.

The damping ratio is defined as the ratio of the actual damping to the damping required for the condition that divides oscillatory from nonoscillatory transients. Draper and Bently (1940) intioduced the use of the undamped natural frequency and the damping ratio in their analysis of instrument performance. They defined the reciprocal of the damping constant as the characteristic time. Either the characteristic time and the damping ratio or the undamped natural frequency and the damping ratio may be used to define the response of physical systems described by linear second-order differential equations such as Eq. (1) and its transform, 
Eq. (3). Draper and Bentley use the characteristic time and the damping ratio for their purposes, but the use of the undamped natural frequency and the damping ratio became generally accepted for use in the analysis of servomechanisms (e.g., Brown and Hall, 1946).

In the complex $s$ plane as shown in Fig. 1, the modulus of the complex variable $s$ (the length of the vector) from the origin to each of the

- poles of the characteristic function of Eq. (3), or the

- roots of the characteristic equation given by Eq. (4),

is equal in magnitude to the undamped natural frequency $2 b / R$. The real component of this vector is equal in magnitude to the damping constant $(2 \mathrm{~b} / \mathrm{R})(\mathrm{b} / \mathrm{a})$. The imaginary component is equal in magnitude to the natural frequency $(2 b / R)\left[-(b / a)^{2}\right]^{1 / 2}$. Inis vector and its real and imaginary components form a right triangle with sides whose lengths are in the proportions

$$
1: b / a:\left[1-(b / a)^{2}\right]^{1 / 2}
$$

or

$$
a: b:\left(a^{2}-b^{2}\right)^{1 / 2}
$$

Therefore, the proportional relations among the damping ratio and the damped and undamped natural frequencies are determined by two parameters: i.e., the compressional-wave velocity a and the shear.wave velocity $b$.

In electrical and mechanical systems, the damping ratio can be less than, equal to, or greater than unity. With these respective values of the damping ratio, the systems are underdamped, critically damped, or overdamped. The elastic system described by Eqs. (3) and (4) is underdamped because Poisson's ratio $v$ for elastic solid is limited to the range of values $0<1<1 / 2$; thus the damping ratio $\zeta$ is restricted to the following range of values:

$$
0<\zeta=b / a=[\mu /(\lambda+2 \mu)]^{1 / 2}=[(1-2 \nu) / 2(1-\nu)]^{1 / 2}<2^{-1 / 2}
$$

The relation between $\zeta$ and $\nu$ is illustrated in Fig. 2 .

\section{CHARACTERISTIC FREQUENCIES}

The Laplace transform may be replaced by the Fourier transform by letting the real component of the complex variable s approach zero so that $s \rightarrow i \omega$ (Gardner and Barnes, pp. 99-107). Then Eq. (3) becomes

$$
-\left(\frac{4 \mu}{R^{3}}\right) \frac{\hat{\mathbf{X}}(\mathrm{i} \omega)}{\hat{\mathbf{P}}(i \omega)}=\left[1+i\left(\frac{R \omega}{\mathrm{a}}\right)-\left(\frac{\mathrm{R} \omega}{2 \mathrm{~b}}\right)^{2}\right]^{-1}
$$

Inspection of the right side of Eq. (6) gives two convenient definitions for a characteristic dimensionless frequency, i.e., $R \omega / a$ and $R \omega / 2 b$. The former was used by Blake (1952), Herbst et al. (1961), Meyer (1964), and others. The latter was used by Gurvich (1965) and Rodean (1971). These two dimensionless frequencies have equal numerical values if $a=2 b$, i.e., if Poisson's ratio is equal to $1 / 3$ [see Eq. (5) and Fig. 2]. 


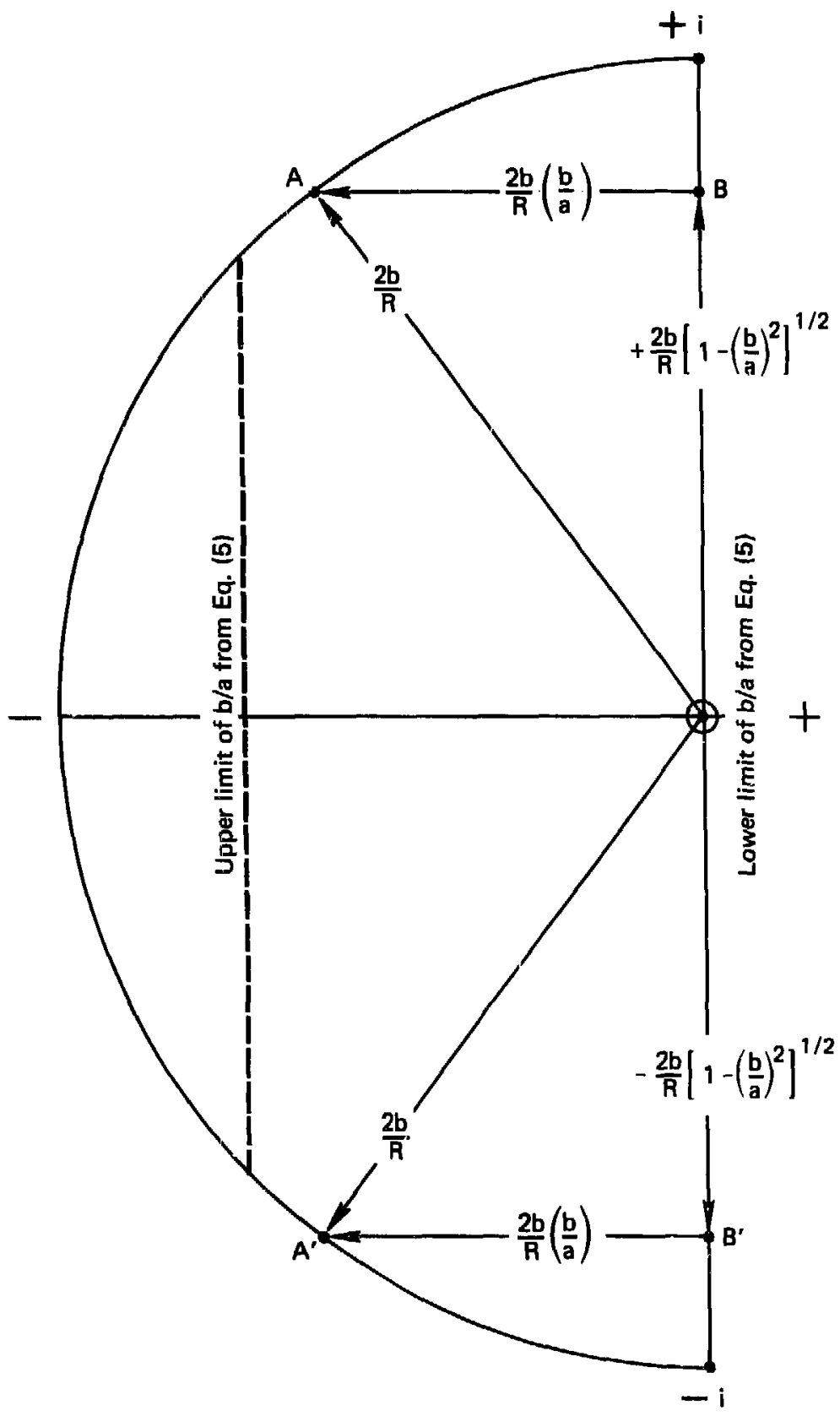

FIG. 1. Poles of the ctaracteristic function [Eq. (3)] and roots of the characteristic equation $[E q .(4)], s=(2 b / R)\left\{-(b / a) \pm\left[\left[1-(b / a)^{2}\right]^{1 / 2}\right\}\right.$. Note that the proportions of the sides of the right triangles $O A B$ and $O A^{\prime} B^{\prime}$ are $a: b:\left(a^{2}-b^{2}\right)^{1 / 2}$. 


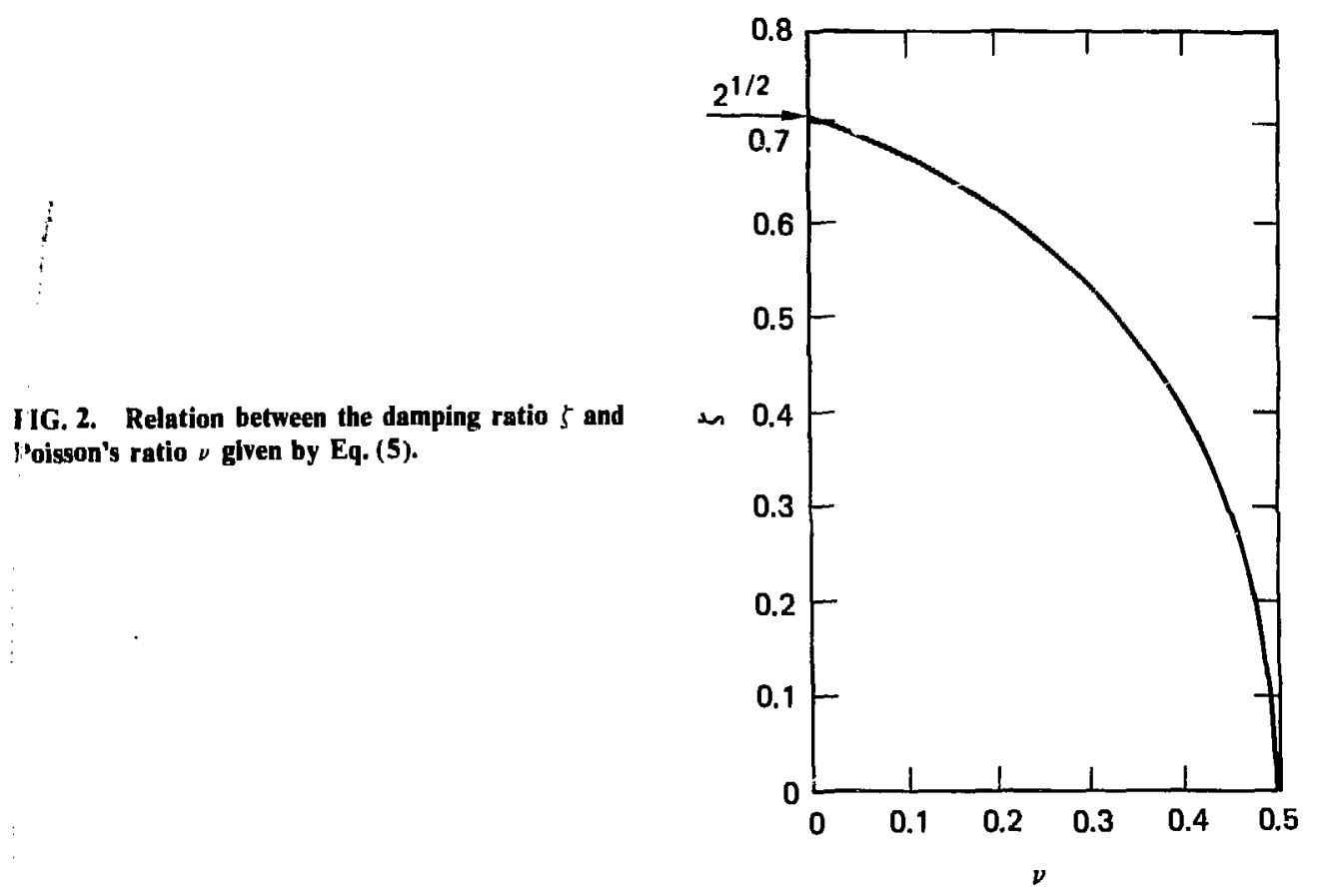

It may seem more appropriate to use $\mathrm{R} \omega / \mathrm{a}$ than $\mathrm{R} \omega / 2 \mathrm{~b}$ as the characteristic dimensionless frequency, because compressional waves, not shear waves, are described by solutions of Eq. ( 1 ) and its transforms, Eqs. (3) and (6). Blake showed that the normalized specific acoustic radiation resistance for a spherical compressional wave source in an elastic solid is identical to that in an elastic fluid and that it is a function of $\mathrm{R} \omega / \mathrm{a}$ alone, i.e., $(\omega \mathrm{R} / \mathrm{a})^{2}\left[1+(\omega \mathrm{R} / \mathrm{a})^{2}\right]^{-1}$. He also showed that the specific acoustic radiation reactance is reduced to that for a fluid if $\mu \rightarrow 0$, i.e., $(\omega R / a)\left[1+(\omega R / a)^{2}\right\}^{-1}$. Blake's solution for the radiation resistance illustrates one case in which $R \omega / a$ is the appropriate dimensionless frequency; other cases are shown later in the discussion of the seismic radiation field, where the appropriate dimensionless frequency is R $\omega / \mathrm{a}$. Blake's solution for the radiation reactance with $\mu \rightarrow 0$ shows that $R \omega / a$ is more appropriate than $R_{\nu} / 2 b$ for analysis of fluid-like elastic solids because $b \rightarrow 0$ as $\mu \rightarrow 0$. However, there are many other cases wliere the use of $\mathbf{R} \omega / 2 b$ results in more convenient and compact mathematical relations that more clearly illustrate the physical principles involved. This is because the undamped natural frequency $R / 2 b$ is a fundamental somponent in the roots of the characteristic equation for spherical radiation in an elastic solid [Eq. (4)] Therefore, the reference frequency used in this paper is the characteristic undamped natural frequency:

$$
\omega_{0}=2 b / R
$$

From Eqs. (4), (5), and (7), the characteristic natural frequency is

$$
\omega_{n}=\omega_{0}\left(1-\xi^{2}\right)^{1 / 2}
$$

Note that the frequency $\omega_{n} \rightarrow \omega_{0}$ as $\zeta \rightarrow 0$ (Figs. 1 and 3), hence $\omega_{0}$ is called the "undamped natural frequency." According to Eq. (8), the natural frequency exists if $0<\xi<1$. This range of values for the damping ratio is 


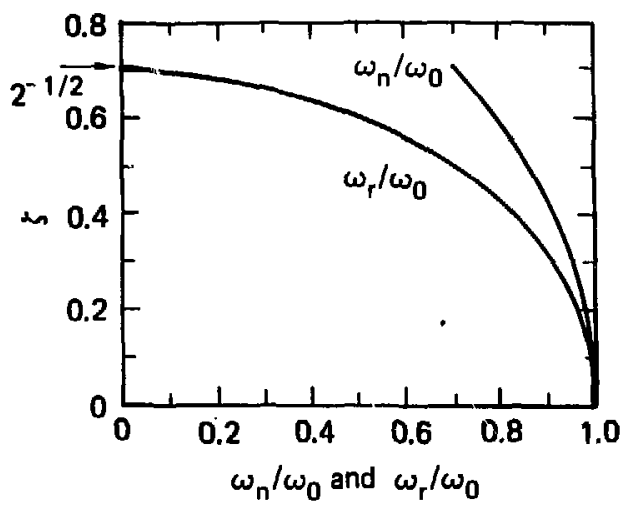

FIG. 3. Relations among the natural, resonant, and undamped natural frequencies $\left(\omega_{n}, \omega_{r}\right.$, and $\omega_{0}$, respectively) as functions of the damping ratio given by Eqs. (8) and (12).

possible for electrical and mechanical systems, but as indicated by Eq. (5), the restrictions on the value for the Poisson's ratio limit the damping ratio for the spherical elastic radiation to $0<5<2^{-1 / 2}$. Therefore, the elastic radiator described by Eqs. (3) and (6) is an underdamped, hence oscillatory, source of compression waves.

Equations (5)-(7) may be combined and the result written in terms of the modulus and argument of a complex variable (Papoulis, 1962, p. 7) as

$$
-\left(4 \mu / \mathbf{R}^{3}\right)[\hat{\mathbf{X}}(i \omega) / \hat{\mathbf{P}}(i \omega)]=A_{D}(i \omega) \exp -i \phi(i \omega)
$$

where the modulus or Fourier amplitude

$$
\left.A_{D}(i \omega)=\mid\left\{\left[1-\left(\omega / \omega_{0}\right)^{2}\right]^{2}+(2\} \omega / \omega_{0}\right)^{2}\right\}^{-1 / 2} \mid
$$

and the argument or phase angle

$$
\phi(i \omega)=\tan ^{-1}\left\{2 \zeta \omega / \omega_{0}\left[1-\left(\omega / \omega_{0}\right)^{2}\right]\right\}
$$

As shown by the values of the first and second partial derivatives of Eq. (10) with respect to $\leftarrow$, $A_{D}(i \omega)$ has a minimum value at $\omega=0$ and a maximum value at the resonant frequency (Gille, Pelegrin, and Decaulne, 1959, pp. 99-101)

$$
\omega_{r}=\omega_{0}\left(1-2 \zeta^{2}\right)^{1 / 2}
$$

The resonant frequency exists only if $0<\xi<2^{-1 / 2}$, the limits defined by Eq. (8), as illustrated in Fig. 3 .

At high frequencies, $A_{\mathrm{D}}(\mathrm{i} \omega) \rightarrow 0$, and $\phi(\mathrm{i} \omega) \rightarrow \pi$. Therefore, the physical system described by Eqs. (3) and (6) acts as a low-pass filter (Gille et al., pp. 103-106; Papoulis, pp. 117-118). There is no standard definition of the cutoff frequency of a low-pass filter, but one definition is the frequency $\omega_{c}$ at which the phase angle is equal to $\pi / 2$ (Gille et al., pp. 103-104)

$$
\omega_{c}=\omega_{0} .
$$


TABLE 1. Values of $A_{D}(i \omega)$ and $\phi(i \omega)$ for several frequencies.

\begin{tabular}{|c|c|c|}
\hline$\omega$ & $A_{D}(i \omega)$ from Eq. (10) & $\phi(i \omega)$ from Eq. (11) \\
\hline $\begin{array}{l}0 \\
\omega_{r}=\omega_{0}\left(1-2 \zeta^{2}\right)^{1 / 2} \\
\omega_{n}=\omega_{0}\left(1-\zeta^{2}\right)^{1 / 2} \\
\omega_{c}=\omega_{0} \\
\omega \rightarrow \infty\end{array}$ & $\begin{array}{l}1 \\
{\left[2 \xi\left(1-\xi^{2}\right)^{1 / 2}\right]^{-1}} \\
{\left[2 \xi\left(1-3 \xi^{2} / 4\right)^{1 / 2} 1^{-1}\right.} \\
(2 \xi)^{-1} \\
\left(\omega_{0} / \omega\right)^{2} \rightarrow 0\end{array}$ & $\begin{array}{l}0 \\
\tan ^{-1}\left[\left(1-25^{2}\right)^{1 / 2} / \zeta\right] \\
\tan ^{-1}\left[2\left(1-\zeta^{2}\right)^{1 / 2} / \zeta\right] \\
\pi / 2 \\
\tan ^{-1}\left(-25 \omega_{0} / \omega\right)-\pi\end{array}$ \\
\hline
\end{tabular}

The definitions of the above characteristic frequencies and corresponding values of $A(i \omega)$ and $\phi(i \omega)$ from Eqs. (10) and (11), respectively, are given in Table 1 , together with values of $A(i \omega)$ and $\phi(i \omega)$ for $\omega=0$ and $\omega \rightarrow \infty$. Solutions for $A(i \omega)$ and $(i \omega)$ are plotted as functions of $\omega / \omega_{0}$ and 5 in Figs. 4 and 5, respectively.

FIG. 4. Fourier amplitude [Eq. (10)] of the transform equation for $-\left(4 \mu / R^{3}\right)[X(i \omega) / P(i \omega)]$ [Eq. (9)] as a function of the frequency ratio $\omega / \omega_{0}$ with the damping ratio $\zeta$ as a parameter. The maximum value for $\zeta$ is $\bar{i}-1 / 2 ; 0.1$ is a minimum practical value.

FIG. 5. Phase rigle [Eq. (11)] of the transform equation for $-\left(-4 / \mathbf{R}^{3}\right)[X(i \omega) / \mathbf{P}(i \omega)]\left[E_{q} \cdot(9)\right]$ as a function of the frequency ratio $\omega / \omega_{0}$ with the damping ratio $\zeta$ as a parameter. The maximum value for $\zeta$ is $2^{-1 / 2} ; 0.1$ is a minimum practical value.
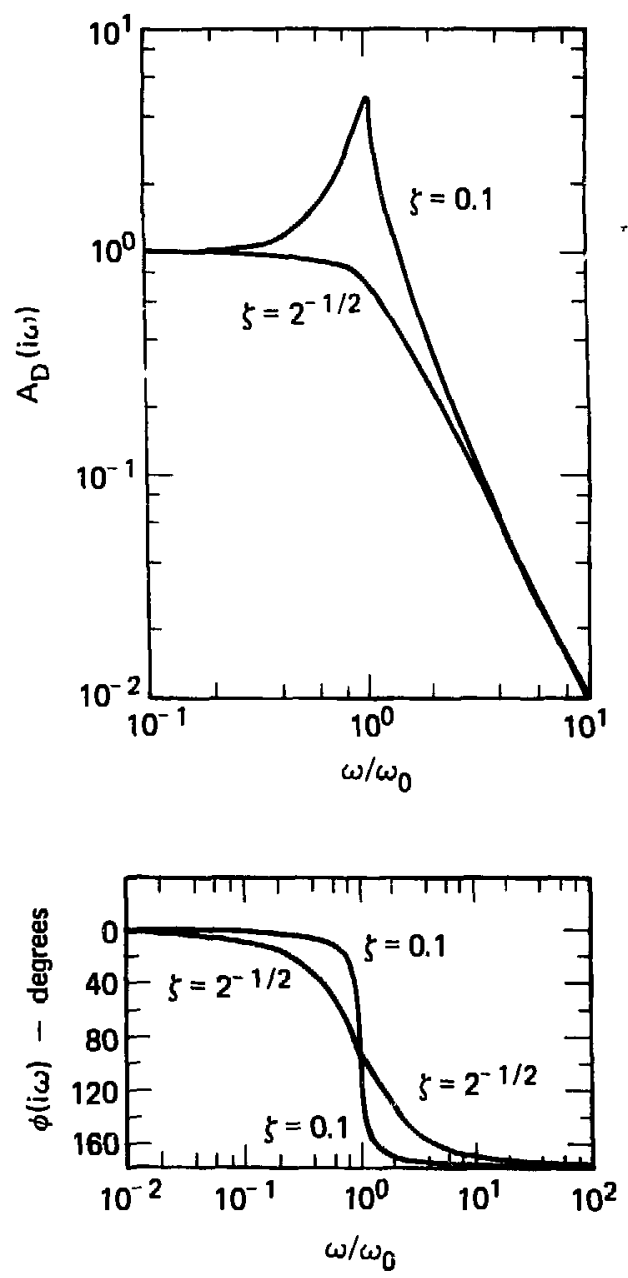


\section{CHARACTERISTIC PERIODS AND WAVELENGTHS}

The period $T_{0}$ corresponding to the frequency $\omega_{0}$ as defined by Eq. (7) is equal to the time required for a shear wave to travel halfway around the surface of a sphere with radius $\mathbf{R}$ :

$$
T_{0}=\pi R / b=\Lambda_{0} / b
$$

where $\Lambda_{0}$ is the characteristic wavelength of the shear wave. The period $T_{n}$, corresponding to $u_{n}$ as defined by Eq. (8) and the corresponding wavelength $\Lambda_{n}$, are related to $T_{0}$ and $\Lambda_{0}$ as follows:

$$
\begin{aligned}
& T_{n}=T_{0}\left(1-\zeta^{2}\right)^{-1 / 2}, \\
& \Lambda_{n}=\Lambda_{0}\left(1-\zeta^{2}\right)^{-1 / 2} .
\end{aligned}
$$

For $\zeta>0, T_{n}>T_{0}$ and $\Lambda_{n}>\Lambda_{0}$. With the surface of the spherical cavity in free radial oscillation at the natural frequency $\omega_{n}$, there is more than sufficient time during each oscillation period $T_{n}$ for communication by means of shear waves between all points on the surface. The time $T_{0}$ is exactly sufficient for such communication for oscillation at the undamped natural frequency $\omega_{0}$.

However, none of the above equations describes the propagation of shear waves in any direction; the equations in this paper describe different aspects of the generation and propagation of spherically symmetric compressional or dilatational waves. The same kind of physical relation-or coincidence-as stated by Eq. (14) is given by Eq. (27), which defines a transition in the radial stress field outside the cavity. The significance of the relations given by Eq. (14) is discussed further in connection with Eq. (27).

\section{DAMPING}

The electrical analog of the cavity-elastic-solid system described by Eqs. (3)-(11) is a resistanceinductance-capacitance circuit, and its mechanical analog is a dashpot-spring-mass system. The oscillations of these electrical and mechancial systems are damped because energy is dissipated in their resistors and dashpots, respectively. However, Eqs. (3)-(11) represent an ideal elastic system without energy dissipation. The oscillations are damped because energy is radiated away from the source. It is possible for an electrical or a mechanical system to be overdamped $(\zeta>1)$ or critically damped $(\zeta=1)$, but the ideal elastic system described by Eqs. (3)-(11) is underdamped $\left(0<\zeta<2^{-1 / 2}\right)$ because of the restrictions on the value of Poisson's ratio $(0<y<1 / 2)$ as shown by Eq. (5). The damping approaches zero if the :lastic solid tends to become like an elastic fluid $(\nu \rightarrow 1 / 2$ with $\lambda>0$ and $\mu \rightarrow 0)$. The damping approaches the maximum value if the elastic solid tends to become very rigid $(\nu \rightarrow 0$ with $\lambda>0$ and $\lambda / \mu \rightarrow 0)$.

In anelastic wave propagation, the decay of the wave amplitude as a function of time at a fixed point in space has the form $\exp -\omega \tau / 2 Q$, where $Q$ is the specific attenuation or dissipation function (Knopoff, 1964). From Eqs. (4), (5), and (7), the damping of the cavity-elastic-solid system has the form $\exp -\omega_{0} \zeta \tau$. With $\omega=\omega_{0}$, the apparent specific dissipation function for the elastic system described by Eqs. (3)-(II) is

$$
Q=1 / 2 \zeta
$$

From Eqs. (10) and (12), the resonance ratio or peak value of magnification (Gille et al., pp. 99-101) is approximately equal to $Q$ for small values of $\zeta$

$$
A_{D}(i \omega)_{\omega=\omega_{r}} / A_{D}(i \omega)_{\omega=0}=\left[2 \zeta\left(1-\zeta^{2}\right)^{1 / 2}\right]^{-1}
$$


Gille et al. used the symbol $Q$ for the quantity defined by Eq. (18), but they did not identify $Q$ as the specific dissipation factor. They noted that the resonance ratio is greater than unity only if $\zeta<2^{-1} 2$, as is apparent from Eq. (18). Such resonance exists for the elastic system described by Eqs. (3)-(11) because $0<:<2^{-1} 2$ from Eq. (5). The resonance ratio defined by Eq. (18) and its relation to $\mathrm{Q}$ defined by Eq. (17) are plotted as functions of $\zeta$ in Fig. 6.

As noted in connection with Eq. (13), Eqs. (3)-(11) describe a system that acts as a low-pass filter. These equations may be converted into those for a band-pass filter by rewriting them in terms of ax $(\tau) / \partial \tau$, the "reduced-velocity potential" whose Fourier transform is $\omega \hat{\mathrm{X}}(\mathrm{i} \omega)$. Then Eqs. (9) and (10) may be rewritten as

$$
\begin{aligned}
& \left.-\left(\frac{i \omega}{\omega_{0}}\right)\left(\frac{4 \mu}{R^{3}}\right)\left[\frac{X(i \omega)}{\hat{P}(i \omega)}\right]=A_{v}(i \omega) \exp -i \mid \dot{\phi}(i \omega)-\pi / 2\right], \\
& A_{v}(i \omega)=\left|\left(\omega / \omega_{0}\right)\left\{\left[1-\left(\omega / \omega_{0}\right)^{2}\right]^{2}+\left(2 \zeta \omega / \omega_{0}\right)^{2}\right\}^{-1 / 2}\right|
\end{aligned}
$$

The relation for $\phi(\mathrm{i} \omega)$ is given by Eq. (11). The system studied by Gurvich was based on relations for the farfield displacement for a sinusoidal function of $\mathrm{P}(\tau)$, and his equation for the Fourier amplitude of the far-field displacement is similar to Eq. (20). The Fourier amplitude [Eq. (20)] has a maximum value of $1 / 2 \zeta$ at the resonant frequency, $\omega_{\mathrm{r}}=\omega_{0}$ in this case, and is approximately symmetrical about $\omega_{i}=\omega_{0}$ in the $A_{v}$ (ici) vs $\psi^{\prime} \omega_{0}$ plane, as shown in Fig. 7. It is also shown in this figure that $A_{v}(i \omega)$ is proportional to $u^{+1}$ for $0<u<<u_{0}$

FIG. 6. Apparent specific dissipation function Q[Eq. (17)] and the resonance ratio

$$
A_{\mathbf{D}}(\mathbf{i} \omega)_{\omega=\omega \mathbf{r}} / \mathbf{A}_{\mathbf{D}}(\mathbf{i} \omega)_{\omega=0}
$$

[Eq. (18)] as functions of the damping ratio $\zeta$. Note that the value of the resonance ratio appis;ximates that of $Q$ for small values of 5 .

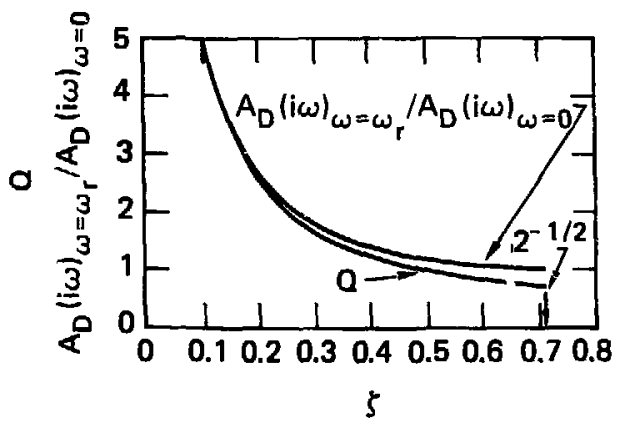

FIG. 7. Fourier amplitude [Eq. (20)] of the transform equation for

$$
-\left(i \omega / \omega_{0}\right)\left(4 \mu / R^{3}[X(i \omega) / \mathbf{P}(i \omega)]\right.
$$

[Eq. (19)] as a function of the frequency ratio $\omega / \omega_{0}$ with the demping ratio 5 as a parameter. The maximum value for $\zeta$ is $2^{-1 / 2} ; 0.1$ is a minimum practical value.

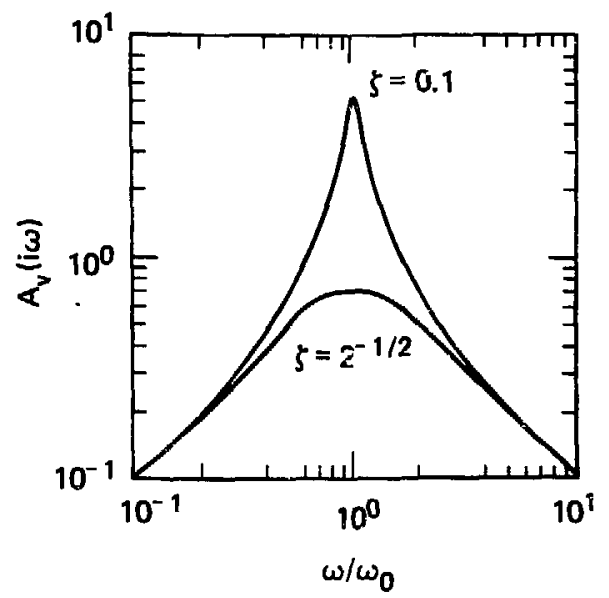




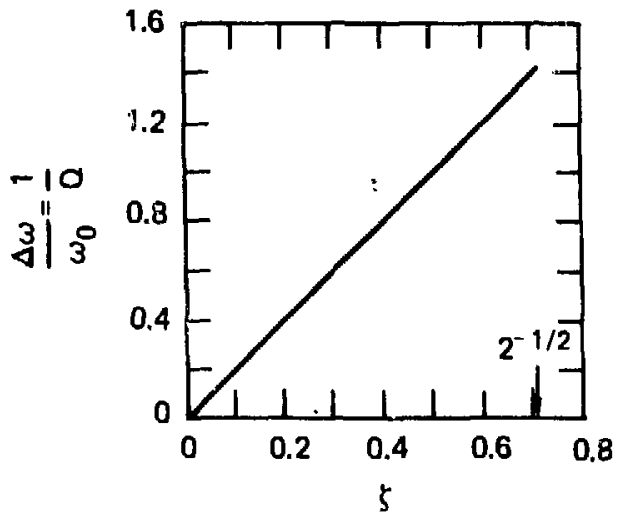

FIG. 8. Bandwidth $\Delta \omega / \omega_{0}$ and the apparent specific dissipation furction $Q$ as functions of the damping ratio givasis by Eq. (22).

and to $\omega^{-1}$ for $\omega_{0}<<\omega<\infty$. The dynamic bandwidth of a bandpass filter is the frequency interval $\Delta \omega$ between the two half-power points where $A_{v}(i \omega) / A_{v}(i \omega)_{\omega=\omega_{0}}=2^{-1 / 2}$ (Papoulis, p. 159). From Eq. (20), for this condition,

$$
\Delta\left(\omega / \omega_{0}=\left[1+2 \zeta^{2}+2 \zeta\left(1+\zeta^{2}\right)^{1 / 2}\right]^{1 / 2}-\left|1+2 \zeta^{2}-2 \zeta\left(1+\zeta^{2}\right)^{1 / 2}\right|^{1 / 2} .\right.
$$

After squaring both sides, Eq. (21) simplifies to

$$
\Delta \omega / \omega_{0}=2 \zeta=1 / Q
$$

and is related to Eq. (17) as shown. Gurvich presented the above relation bet ween $\Delta \omega / \omega_{0}$ and $\zeta$. The relations defined by Eq. (22) are shown in Fig. 8.

The preceding demonstrates that $\omega_{0}$ and $\zeta$ are very useful parameters for describing the radiation of spherical compressional waves from a source in an elastic solid.

\section{SEISMIC RADIATION FIELD}

In the preceding section the source of the seismic radiation, i.e., a spherical cavity in an elastic solid, was the subject of study. In this section the seismic radiation field outside the spherical cavity $(r>R)$ is studied.

\section{CHARACTERISTIC FIELD EQUATIONS}

The Laplace transform of Eq. (1) for the radial stress is similar to Eq. (3)

$$
\left(\frac{r^{3}}{4 \mu}\right) \frac{\hat{\sigma}_{r}(r, s)}{\hat{x}(s)}=\left(\frac{r}{2 b}\right)^{2} s^{2}+\left(\frac{r}{a}\right) s+1 .
$$


Selberg derived equations for both the radial and tangential stresses. The Laplace transform of an equivalent form of his relation for the tangential stress is

$$
\left(\frac{r^{3}}{4 \mu}\right) \frac{\hat{a}_{t}(r, s)}{\hat{X}(s)}=\left(1-2 \zeta^{2}\right)\left(\frac{r}{2 b}\right)^{2} s^{2}-\left(\frac{r}{a}\right) \frac{s}{2}-\frac{1}{2} .
$$

The only displacement in spherical compressional wave motion is radial:

$$
\frac{\mathrm{r}^{2} \hat{\mathrm{u}(\mathrm{r}, \mathrm{s})}}{\hat{\mathrm{X}}(\mathrm{s})}=-\left[1+\left(\frac{\mathrm{r}}{\mathrm{a}}\right) \mathrm{s}\right] .
$$

In Eqs. (23)- (25), $\hat{\mathrm{X}}(\mathrm{s})$ is the driving transform, and $\hat{\sigma}_{r}(r, s), \hat{\sigma}_{t}(r, s)$, and $\hat{u}(r, s)$ are response transforms.

\section{TRANSITIONS BETWEEN NEAR AND FAR FIELDS}

The following discussion is similar to portions of Meyer's comprehensive study of the near and far fields in elastic and viscoelastic media. Many graphical illustrations of near-field $\left(r^{-3}\right.$ or $\left.r^{-2}\right)$ and far-field $\left(r^{-1}\right)$ behavior for several physical parameters (stresses, strains, and radial displacement and its time derivatives) are given in Figs. 1-3, 6b, 7, 16-18, and 21 of Meyer's paper. Mathematical definitions of the transitions between the near and far jieiós for some of these parameters are given in Eqs. (4.2) and (5.1) of that paper. In this paper, additional mathematical definitions of near-to-far-field transitions are given. Equations that appear in either the same or equivalent form in Meyer's paper are identified by an asterisk.

The Fouries transform equivalent of Eq. (23) for the radial stress is similar to Eq. (6):

$$
\left(\frac{r^{3}}{4 \mu}\right) \frac{\hat{a}_{r}(r, i \omega)}{\hat{x}(i \omega)}=1+i\left(\frac{r \omega}{a}\right)-\left(\frac{r \omega}{2 b}\right)^{2} .
$$

For values of $r \omega \rightarrow 0$, or for the static case with $\omega=0$, the radial stress has relatively large values in only the near field:

$$
\left[\left(\frac{R^{3}}{4 \mu}\right)_{\frac{\hat{\sigma}_{r}(r, i \omega)}{\hat{x}(i \omega)}}^{\hat{\hat{n}}(\omega) \rightarrow 0}=\left(\frac{R}{r}\right)^{3} .\right.
$$

For values of $\mathrm{r} \omega>>\mathbf{R} \omega_{0}$ [see Eq. (7)], the radial stress has significantly large values that extend into the far field:

$$
\left[\left(\frac{R^{3}}{4 \mu}\right) \frac{\hat{\sigma}_{r}(r, i \omega)}{\hat{x}_{(i \omega)}}\right]_{r \omega>>R \omega_{0}}=-\frac{R}{r}\left(\frac{\omega}{\omega_{0}}\right)^{2} .
$$

Let the transition between the near and far fields of the radial stress be defined by the condition that the real component (the sum of the near- and far-field terms) of Eq. (26) be equal to zero:

$$
\mathbf{r} \omega=2 \mathrm{~b} \text {. }
$$

Note the similarity between Eqs. (7) and (27). The period corresponding to the frequency as defined by Eq. (27) is equal to the time required for a shear wave to travel halfway around a sphere defined by the radius 
marking the transition between the near and far radial-stress fields. Meyer explained the role of the shearwave velocity in Eq. (27) as follows: "In the stress. . .transition,.. . the stored elastic energy (prevalent in the near field) begins to compare in magnitude with the kinetic energy of the solid. Since no quasi-static volumetric energy can be stored elastically in an infinite sphere under internal pressure, this equivalence expresses itself in tarms of an elastic shear wave length, even though such waves cannot exist in spherically symmetric motions." By analogy, this explanation may also apply to Eqs. (7) and (14). The transitional radial stress in the middle field, from Eqs. (26) and (27), is

$$
\left[\left(\frac{R^{3}}{4 \mu}\right){\frac{\hat{a}_{r}(r, i \omega)}{\hat{x}(i \omega)}}_{r \omega=2 b}=i\left(\frac{R}{r}\right)^{2} \frac{K \omega}{a} .\right.
$$

Note that the transitional radial stress is proportional to $r^{-2}$, intermediate between the relations for the near field $\left(r^{-3}\right)$ and the far field $\left(r^{-1}\right)$.

The Fourier transform for the tangential stress, from Eqs. (5j and (24),

$$
\left(\frac{r^{3}}{4 \mu}\right) \frac{\hat{\sigma}_{t}(r, i \omega)}{\hat{X}(i \omega)}=-\left[\frac{1}{2}+\frac{i}{2}\left(\frac{r \omega}{a}\right)+\left(1-2 \zeta^{2}\right)\left(\frac{r \omega}{2 b}\right)^{2}\right] \text {. }
$$

Equation (28) has near- and far-field properties similar to those of Eq. (26) that are described above. However, the transition between the near and far fields of the tangential stress cannot be defined by the condjtion that the real component of Eq. (28) be equal to zero, as is the case with Eq. (26). As an equivalent, let the transition between the near and far fields of the tangential stress be defined by the condition in which the ratio of the imaginary component (the transitional field) to the real component (the sum of the near- and fas-field terms) of Eq. (28) is a maximum:

$$
r \omega=2 b\left[2\left(1-2 \zeta^{2}\right)\right]^{-1 / 2} .
$$

For the condition defined by Eq. (29), the near- and far-field terms in Eq. (28) are equal in magnitude.

The maximum shestr stress (Jaeger and Cook, 1971, p. 22) is

$$
\sigma_{\mu}=\frac{1}{2}\left(\sigma_{r}-\sigma_{\tau}\right)
$$

From Eqs. (26), (28), and (30),

$$
\left(\frac{r^{3}}{4 \mu}\right) \frac{\hat{\sigma}_{\mu}(r, i \omega)}{\hat{x}(i \omega)}=\frac{1}{4}\left[3+3 i\left(\frac{r \omega}{a}\right)-\left(\frac{r \omega}{2}\right)^{2}\right] .
$$

In this case the appropriate dimensionless frequency is obviously $r \omega / \mathrm{a}$, not $\tau \omega / 2 b$.

$n$. in the case of the radial stress, let the transition between the near and far fielưs of the maximum shear stress ive defined as the condition in which the real component of Eq. (3I) is equal to zero:

$$
\mathrm{r} \omega=\mathrm{a}(3)^{1 / 2} \text {. }
$$

The mean normal stress (or pressure) determines uniform compression or dilatation and is defined (Jaeger and Cook, p. 32) as

$$
\sigma_{m}=\frac{1}{3}\left(\sigma_{r}+2 \sigma_{t}\right) \text {. }
$$

The bulk modulus is defined as

$$
\mathbf{k}=\lambda+2 \mu / 3 .
$$


From Eqs. (26), (28), (33), and (34),

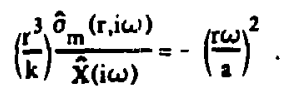

There is no near field in this $c$ se because the mean stress varies as $r^{-1}$ everywhere, in contrast to the radial. 'angential, and maximum shear stresses. As for the maximum shear stress, the appropriate dimensionless frequency is $r \omega / \mathrm{a}$, not $\mathrm{r} \omega / 2 \mathrm{~b}$.

The Fourier transform for the radial displacemenh, from Eq. (25), is

$$
r^{2} \frac{\hat{u}(r, i \omega)}{\hat{X}(i \omega)}=-\left[1+i\left(\frac{r \omega}{a}\right)\right] .
$$

This equation is also applicable to the tangential strain $u / r$. It call be modified to give the transform for the radial velocity and radial acceleration, respectively:

$$
\begin{aligned}
& r^{2} \frac{\partial \hat{u}(r, i \omega) / \partial \tau}{i \omega \hat{X}(i \omega)}=-\left[1+i\left(\frac{r \omega}{a}\right)\right], \\
& r^{2} \frac{\partial^{2} \hat{u}(r, i \omega) / \partial \tau^{2}}{\omega^{2} \hat{X}(i \omega)}=\left[1+i\left(\frac{r \omega}{a}\right)\right] .
\end{aligned}
$$

Let the transition between the near and far fields of the

radial displacement [Eq. (36)],

ingential strain [Eq. (36)],

radial velocity $[\mathrm{Eq} .(3 i)$ and

radial acceleration [Eq. $(, 8)$ !

be defined as the condition in which the real and imaginary components (the near- and far-field terms, respectively) of these equations are equal in value. Then

$$
r \omega=\mathbf{a} \text {. }
$$

It is clear that $\mathrm{r} \omega / \mathrm{a}$, not $\mathrm{r} \omega / 2 \mathrm{~b}$, is the appropriate dimensionless frequency for the radial displacement, velocity, and acceleration, and for the tangential strain.

The radial strain from Meyer, is

$$
\frac{\partial u}{\partial r}=\frac{u}{r}+\frac{\sigma_{\mu}}{\mu}
$$

From Eqs. (31), (36), and (40),

$$
\mathrm{r}^{3} \frac{\partial \hat{\mathrm{u}}(\mathrm{r}, \mathrm{i} \omega) / \partial \mathrm{r}}{\hat{\mathrm{x}}(\mathrm{i} \omega)}=2+2 \mathrm{i}\left(\frac{\mathrm{r} \omega}{\mathrm{a}}\right)-\left(\frac{\tilde{r} \omega}{\mathrm{a}}\right)^{2} .
$$

As in the cases of the radial and maximum shear stresses, let the transition between the near and far fields of the radial strain be defined as the condition in which the real component of Eq. (40) is equal to zero:

$$
r c^{:}:-\mathrm{a}(2)^{1 / 2} \text {. }
$$

It is clear that there is no single definition in terms of $\mathrm{r} \omega$ for the transition between the near and far fields of spherical compressional-wave radiation. The definitions vary from parameter to parameter. The 
TABLE 2. Definitions of transitions between nedr ard far firlds in opherical compressiond-wave radiation.

\begin{tabular}{|c|c|c|}
\hline Item & $\mathrm{Eq}$ & Value of rw \\
\hline Redial stress & (27) & $2 b$ \\
\hline Tengencid strea & (29) & $2 b\left[2\left(1-2 \xi^{2}\right)\right]^{-1 / 2}$ \\
\hline Maximum thear atrea & (32) & $(3)^{1 / 2}$ \\
\hline Rediel strain & (42) & $=(2)^{1 / 2}$ \\
\hline $\begin{array}{l}\text { Radial diplecement, velocity, } \\
\text { and acceleraxion, tangential uruin }\end{array}$ & (39) & $=$ \\
\hline
\end{tabular}

definitions involve the shear-wave velocity in some cases and the compressional-wave veivity in others. These are summarized in Table 2.

The moduli of the complex Fourier transforms of the spherical compressional-wave field parameters defined by Eqs. (26), (28), (31), (35)-(38), and (41) are presented in dimensionless form in Table 3. These moduli are plotled in Figs. 9-14 as functions of $r / R$ with $u^{\prime} / \omega_{0}$ and $\zeta$ or $R \omega / a$ as parameters. Note that there are strong relations between the near field and low frequencies and the far field and high frequencies. Other relations, such as those for relative phase in the near and far fields, are given by Meyer.

TABLE 3. Moduli of Fouriet transforms for spherical compressional-wave radiation. These are plutted in Figs. 9-14 as functions of $r / R$ with $\omega / \omega_{0}$ and $\zeta$ or $R \cdot \omega / 2$ as parameters.

\begin{tabular}{|c|c|c|c|c|}
\hline Item & Fq & Parmineter & Modull (absolute veluea) & Fig. \\
\hline $\begin{array}{l}\text { Radid } \\
\text { strets }\end{array}$ & $(;, 6)$ & $\frac{R^{3} \hat{\sigma}_{r}(r, i \omega)}{4 \mu \hat{X}(i \omega)}$ & $\left\{\left[\left(\frac{R}{r}\right)^{3}-\frac{R}{r}\left(\frac{\omega}{\omega_{0}}\right)^{2}\right]^{2}+4\left(\frac{R}{r}\right)^{4}\left(\frac{\omega_{r}^{r}}{\omega_{0}}\right)^{2}\right\}^{1 / 2}$ & 9 \\
\hline $\begin{array}{l}\text { Tangentid } \\
\text { streas }\end{array}$ & (28) & $\frac{R^{3} \hat{\sigma}_{t}(r, i \omega)}{4 \mu \hat{X}(i \omega)}$ & $\left.\left\{i\left(\frac{R}{r}\right)^{3}+\left(1-2 \zeta^{2}\right)\left(\frac{R}{r}\right)\left(\frac{\omega}{\omega_{0}}\right)^{2}\right]^{2}+\left(\frac{\pi}{4}\right)^{4}\left\{\frac{\omega_{\gamma}}{\omega_{0}}\right)^{2}\right\}^{1 / 2}$ & 10 \\
\hline $\begin{array}{l}\text { Muximum thear } \\
\text { stres: }\end{array}$ & (31) & $\frac{\mathrm{R}^{3} \hat{\sigma}_{\mu}(\mathrm{r}, \mathrm{i} \omega)}{4 \mu \hat{\mathrm{x}}(\mathrm{i} \omega)}$ & $\frac{1}{4}\left\{\left[3\left(\frac{R}{r}\right)^{3}-\frac{R}{r}\left(\frac{R \omega}{a}\right)^{2}\right]^{2}+9\left(\frac{R}{r}\right)^{4}\left(\frac{R \omega}{a}\right)^{2}\right\}^{1 / 2}$ & 11 \\
\hline $\begin{array}{l}\text { Mean normal } \\
\text { stress }\end{array}$ & (35) & $\frac{\mathrm{R}^{3} \hat{\sigma}_{\mathrm{m}}(\mathrm{r}, \mathrm{i} \omega)}{\mathrm{k} \hat{\mathrm{X}}(\mathrm{i} \omega)}$ & $\frac{R}{r}\left(\frac{R \omega}{a}\right)^{2}$ & 12 \\
\hline $\begin{array}{l}\text { Rudin } \\
\text { strain }\end{array}$ & (41) & $\frac{\mathbf{R}^{3} \partial \hat{u}(\mathbf{r}, \mathbf{i} \omega) / \partial \mathbf{r}}{\hat{\mathbf{X}}(\mathbf{i} \omega)}$ & $\left\{\left[2\left(\frac{\mathrm{R}}{\mathrm{r}}\right)^{3}-\frac{\mathrm{R}}{\mathrm{r}}\left(\frac{\mathrm{R} \omega}{\mathrm{a}}\right)^{2}\right]^{2}+4\left(\frac{\mathrm{R}}{\mathrm{r}}\right)^{4}\left(\frac{\mathrm{R} \omega}{\mathrm{a}}\right)^{2}\right\}^{1 / 2}$ & 13 \\
\hline $\begin{array}{l}\text { Redial } \\
\text { displecement }\end{array}$ & (36) & $\frac{\mathbf{R}^{2} \mathbf{u}(r, i \omega)}{\hat{\mathbf{x}}(i \omega)}$ & $\frac{\mathrm{R}}{\mathrm{r}}\left[\left(\frac{\mathrm{R}}{\mathrm{r}}\right)^{2}+\left(\frac{\mathrm{R} \omega}{\mathrm{a}}\right)^{2}\right]^{1 / 2}$ & 14 \\
\hline $\begin{array}{l}\text { Redial } \\
\text { velocity }\end{array}$ & (37) & $\frac{\mathbf{R}^{2} \partial \hat{\mathbf{u}}(\mathbf{r}, \mathrm{i} \omega) / \partial \tau}{i \omega \hat{\mathrm{X}}(\mathrm{i} \omega)}$ & $\frac{\mathrm{R}}{\mathrm{r}}\left[\left(\frac{\mathrm{R}}{\mathrm{r}}\right)^{2}+\left(\frac{\mathrm{R} \omega}{\mathrm{a}}\right)^{2}\right]^{1 / 2}$ & 14 \\
\hline $\begin{array}{l}\text { Redial } \\
\text { attenuation }\end{array}$ & (38) & $\frac{R^{2} \partial^{2} \hat{\mu}(r, j \omega) / \partial \tau^{2}}{\omega^{2} \hat{X}(i \omega)}$ & $\frac{\mathrm{R}}{\mathrm{r}}\left[\left(\frac{\mathrm{R}}{\mathrm{r}}\right)^{2}+\left(\frac{\mathrm{R} \omega}{\mathrm{a}}\right)^{2}\right]^{1 / 2}$ & 14 \\
\hline
\end{tabular}




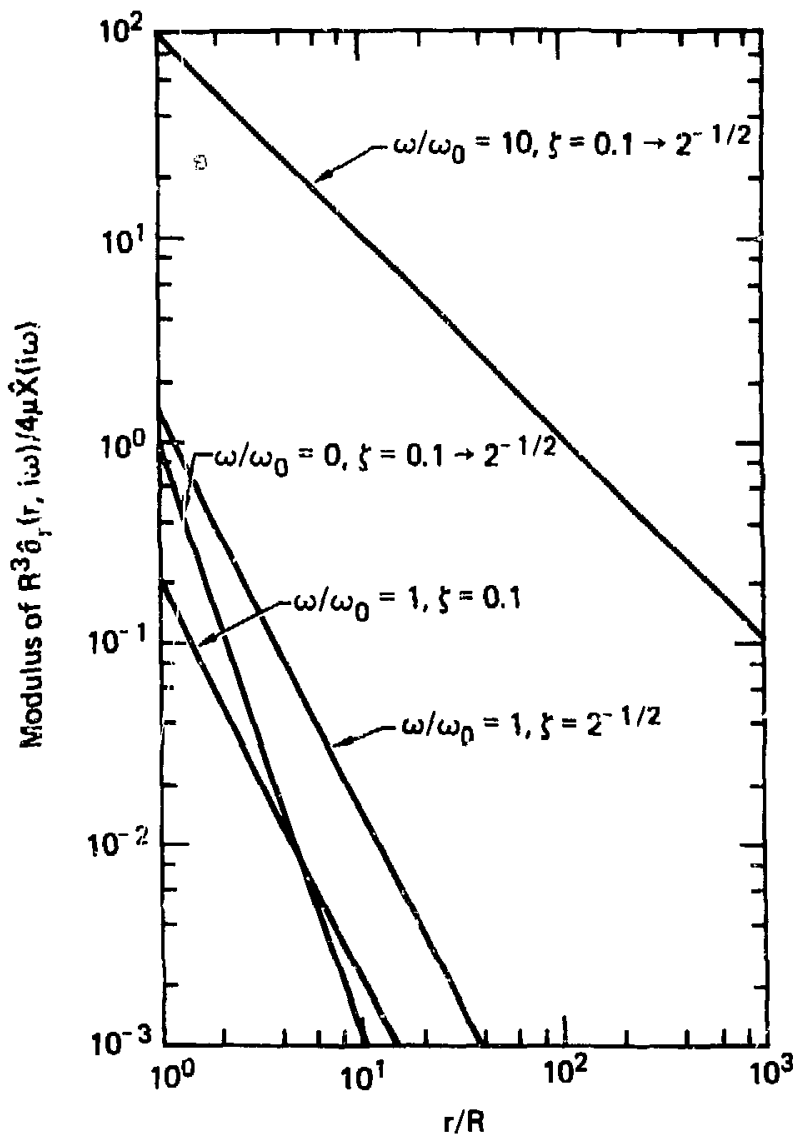

FIG. 9. Mcdulus of the Fourier transform of the radial stress [Eq. (26)] as $\mathrm{g}$ function of $\mathrm{r} / \mathrm{R}$ with $\omega / \omega_{0}$ and $\zeta$ as parameters. The solution for $\omega / \omega_{0}=0$ is independent of $\zeta$ and is the near-field staticstress distribution. For $\omega / \omega_{0}=1$, the condition of $\mathrm{E}_{\mathrm{g}}$. (27) is met and the modulens of $\mathrm{Eq}$. (26) is exactly equal to the imaginary component. Therefore the modulus is directly proportional to $\zeta$ and the stress distribution is that of the intermedinte ficld. For $\omega / \omega_{0}=10$, the efrect of $\zeta$ is negisgible and the stress distribution is that of the far field. 


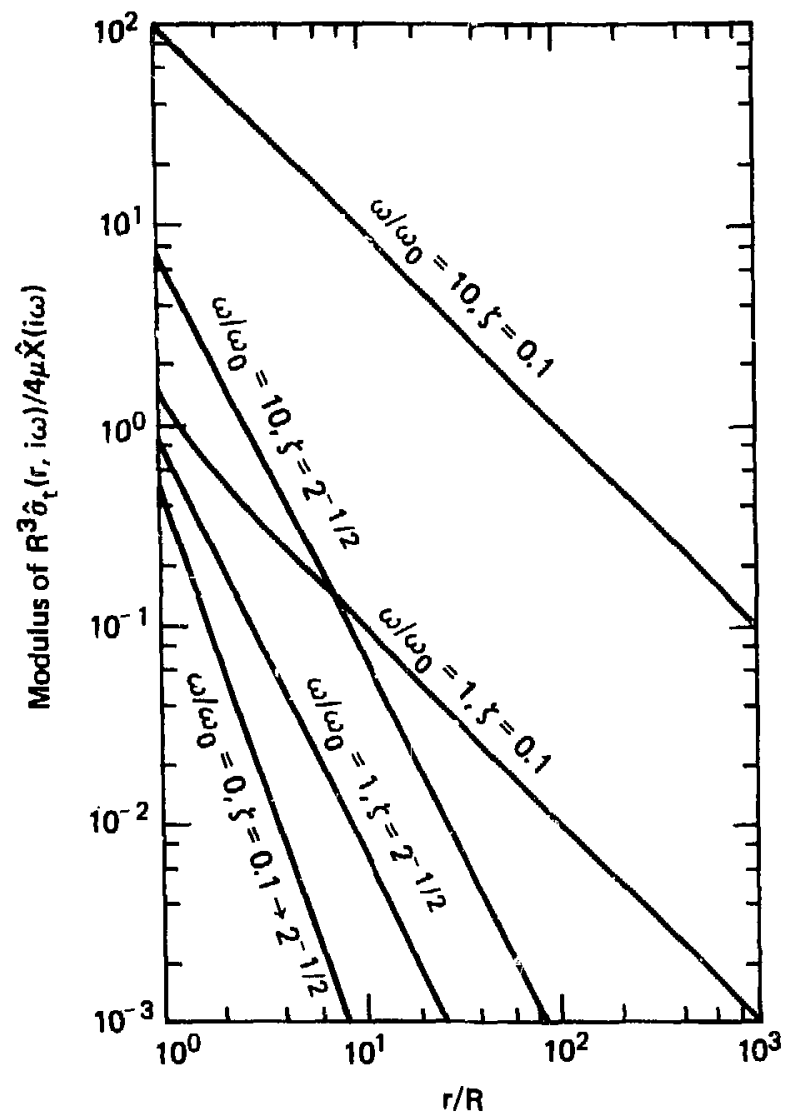

FIG. 10. Modulus of the Fourier transiorm of the tangential stress [Eq. (28)] as a function of $r / R$ with $\omega / \omega_{0}$ and $\zeta$ as parameters. The solution for $\omega / \omega_{0}=0$ is independent of $\zeta$ and is the near-field staticstress distribution. For $\zeta=2^{-1 / 2}$ and $\omega / \omega_{0} \neq 0$, only the near-ficld and the (imaginary) transitional field contribute to the modulus. Hence the intermediate characteristics of the stress field for $\zeta=2^{-1 / 2}$ and $\omega / \omega_{0}=1$ and 10 , with a tendency toward near-field characteristics as $r / R \rightarrow 1$. For $\zeta=0.1$, the stress distribution is characteriatic of the far field for both $\omega / \omega_{0}=1$ and 10 , with tendency toward near-field chanacteristics for $\omega / \omega_{0}=1$ as $r / R \rightarrow 1$. 


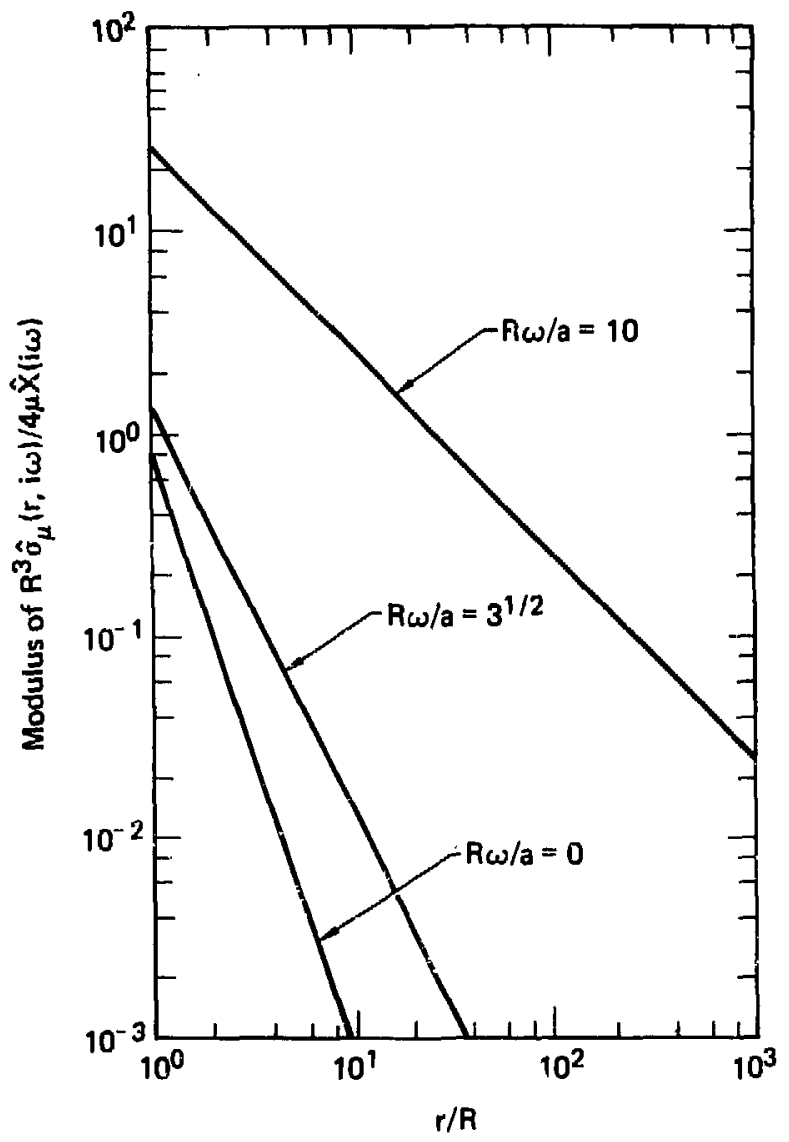

FIG. 11. Modulus of the Fourier transform of the maximum shear stress [Eq. (31)] as a function of $r / R$ with $R \omega /$ a as a parameter. The solution for $\mathbf{R} \omega / \mathrm{a}=\mathbf{0}$ is the statlc near-field stress distribution. For $R \omega / a=3^{1 / 2}$, the condition of Eq. (32) is met and the modulus of Eq. (31) is exactly equal to its imaginary component. Therefore, the stress distribution is that of the intermediate field. For $R \omega / a=10$, the stress distribution is that of the far field. 


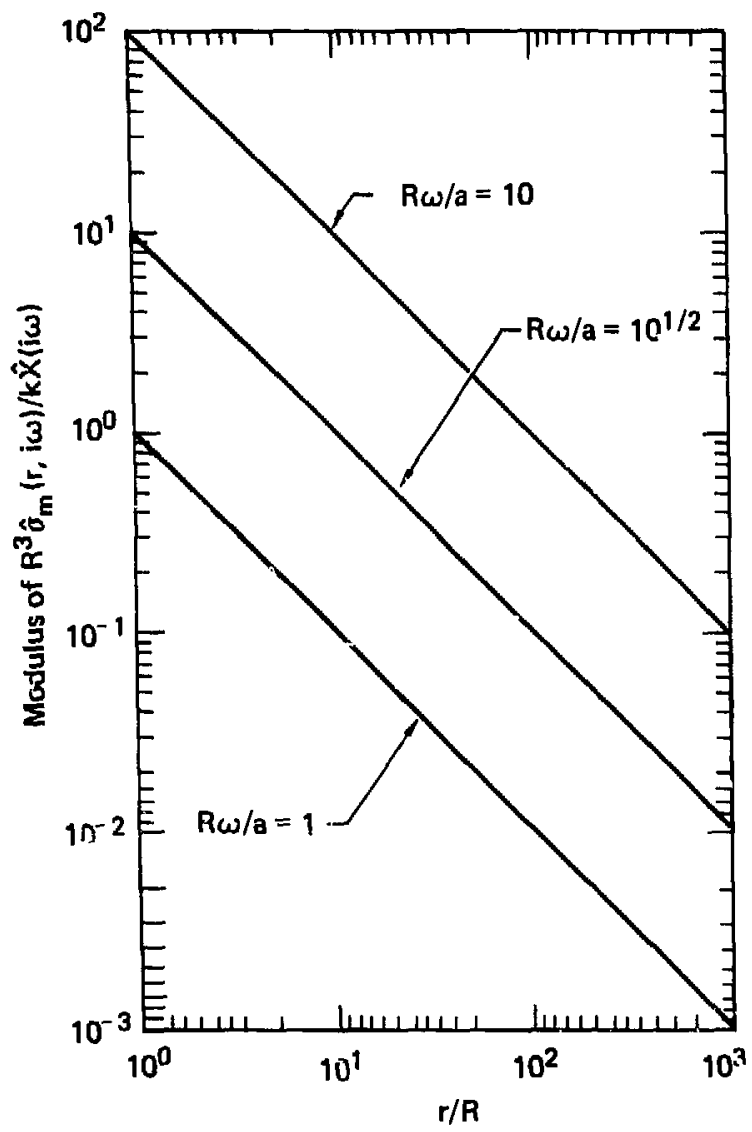

FIG. 12. Modulus of the Fourier transform of the mean normal stress [Eq. (35)] as a function of $r / R$ with $R \omega /$ as a parameter. The modulus is directly proportional to the square of $\mathbf{R} \omega / \mathrm{a}$, hence it is zero if $\omega=0$. The stress distribution is that of only the far field. 


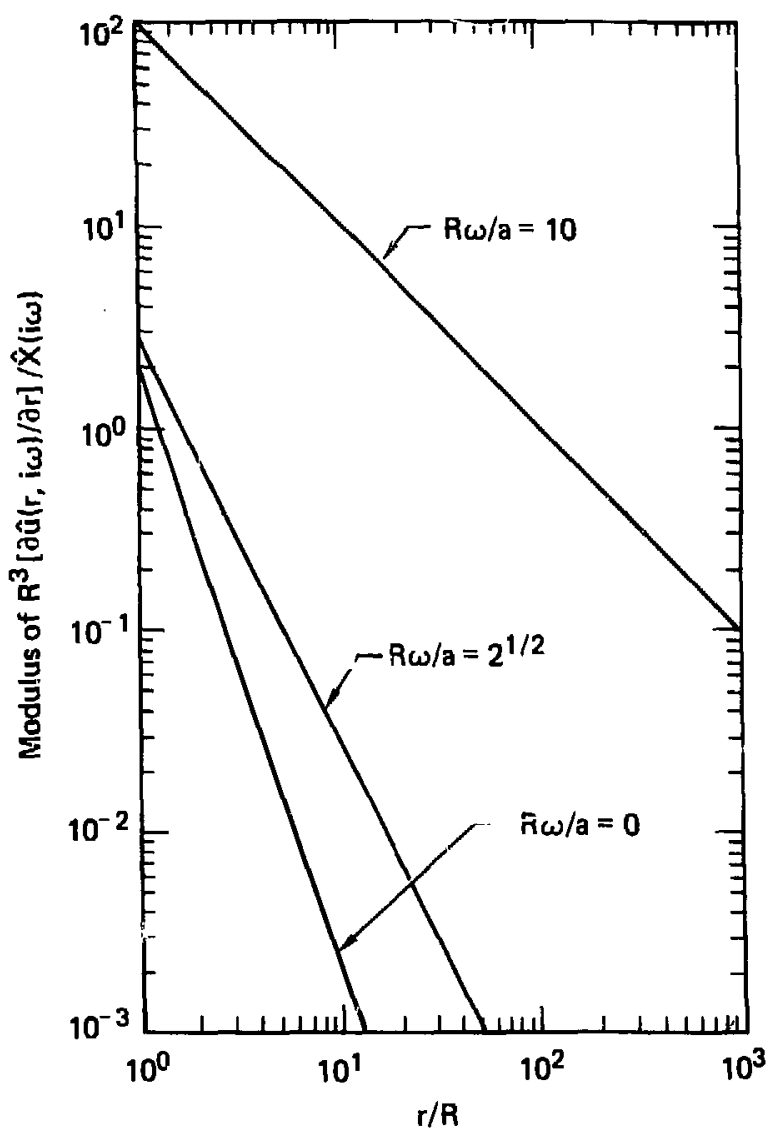

FIG. 13. Modulus of the Fourie transform of the radial strain [Eq. (41)] as a function of $r / R$ with $R \omega /$ a as a parameter. The solution for $\mathbf{R} \omega / \mathrm{a}=\mathbf{0}$ is the static near-field gtrain distribution. For $R \omega / a=2^{1 / 2}$, the condition of Eq. (42) is met and the modulus of Eq. (41) is exactly equal to its imaginary component. Therefore, the strain distribution is that of the intermediate field. For $R \omega / a=10$, the strain distribution is that of the far field. 


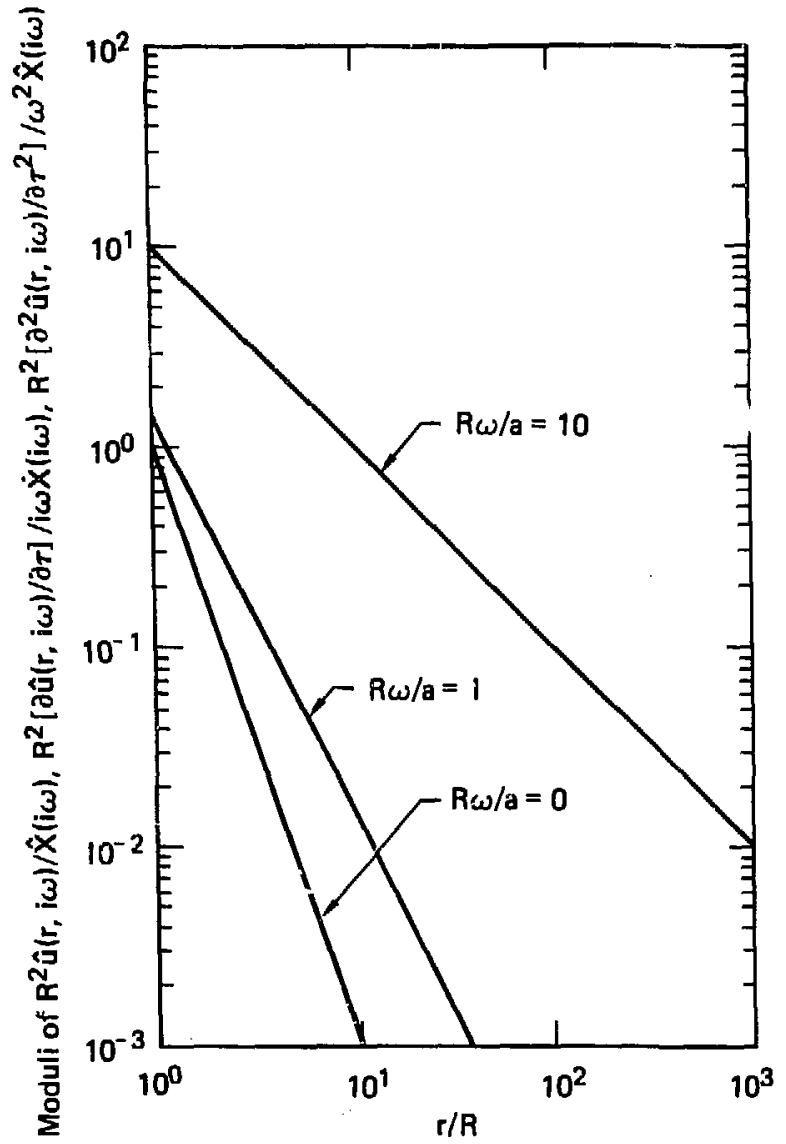

FIG. 14. Modulus of the Fourier transform for the radial displacement [Eq. (36)], radial velocity [Eq. (37)], and radial acceleration [Eq. (38)] as a function of $r / R$ with $R \omega / a$ as a parameter. This figure in also applicable to the tangential strain $u / r$. The solution for $\mathbf{R} \omega / \mathbf{z}=\mathbf{0}$ is the static distribution and is applicable only to the radial displacement and the tangential strain. For $R_{\omega} / \mathbf{m}=1$, the condition of Eq. (39) is met and the real and Imaginary components of Eqs. (36)-(38) are exactly equal to each other. Therefore, the displacement, velocity, acceleration, and tangential strains have distributions characteristic of the intermediate fleld. For $R \omega / a=10$, the distributions - characteristic of the far field. 


\section{TRANSIENT SOLUTIONS FOR SEISMIC-RADIATION SOURCE AND FIELD}

Because of the roots [Eq. (4)] of the characteristic equations from Eq. (3) relating the output $\hat{X}(s)$ to the input $\hat{\mathbf{P}}(\mathrm{s})$, the transient solutions for the

reduced displacement potential [Eq. (3)];

stresses [Eqs. (23), (24), (31), and (35)];

strains [Eqs. (25), (41)];

radial displacement [Eq. (25)]; and

partial derivatives with respect to time of

the reduced displacement potential

and radial displacement;

"I sude damped sinusoidal functions of the form

$$
\left(\exp -\omega_{0} \zeta \tau\right)\left[\sin \left(\omega_{n} \tau-\psi\right)\right] .
$$

The parameters $\zeta, \omega_{0}$, and $\omega_{n}$ are defined by Eqs. (5), (7), and (8), respectively. The phase angle $\psi$ is a function of $\zeta, \omega_{0}$, and parameters associated with the iriput function $P(\tau)$ and the output functions $X(\tau), \sigma_{r}(r, \tau)$, etc. (see, for example, the function-transform pairs 1.301-1.376 on pp. 342-345 of Gardner and Barnes). Damped sinusoidal transient solutions for some outputs (reduced-displacement potential, displacement, velocity, stresses, etc.) have been given by Jeffreys, Kawasumi and Yoshiyama, Blake, Selberg, Rodean, and others. Such transient solutions are not repeated in this paper. Instead, a summary of initial and final values of transient solutions is given.

The initial- and final-value theorems (Gardner and Barnes, pp. 265-269) yield the following respective relations batween the Laplace transform and the initial and final values of the transient solution:

$$
\begin{aligned}
& \lim _{s \rightarrow \infty} \mathrm{sF}(s)=\lim _{\tau \rightarrow 0+} f(\tau), \\
& \operatorname{lims}_{s \rightarrow 0} \mathrm{~T}(s)=\lim _{\tau \rightarrow+\infty} f(\tau) .
\end{aligned}
$$

In the initial-value theorem, $\tau \rightarrow 0+$ means that $\tau>0$ and that the conciition $\tau=0$ is approached from positive, not negative, values of $\tau$. Initial and final values of sejsmic-radiation sources and fields are given in Table 4, asstming a step change in cavity pressure-a good approximation to an explosion.

The following are apparent from Table 4 and Figs. 9-14:

The initial value of the transient solution is related to the high-frequency far-field characteristics of the corresponding Fourier transforms.

The final value of the transient solution is related to the zero-frequency near-field characteristics of the corresponding Fourier transforms.

From Table 4, it is clear that the parameters determining the initial and final values of the 12 listed terms are the

step change in cavity pressure $P_{0}$, cavity radius $R$,

undamped natural frequency of the cavity $\omega_{0}$,

damping ratio of the medium $\zeta$, shear modulus of the medium $\mu$, bulk modulus of the medium $k$, and the radial coordinate $r$. 
TABLE 4. Seismic radiation sources and fielda for $\tau \rightarrow 0+$ and $\tau \rightarrow \infty$ for a step change in cavity pressure $\widehat{\mathbf{P}}(\mathrm{s})=\mathrm{F}_{0} / \mathrm{s}$.

\begin{tabular}{|c|c|c|c|c|}
\hline \multirow[b]{2}{*}{ Item $^{2}$} & \multirow[b]{2}{*}{ Eq. } & \multirow[b]{2}{*}{ Symbol } & \multicolumn{2}{|c|}{ Value } \\
\hline & & & $\tau \rightarrow 0+$ & $r \rightarrow \infty$ \\
\hline RDP & $(3),(5),(7)$ & $\frac{\mathbf{X}(\tau)}{\mathrm{R}^{3}}$ & 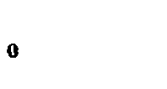 & $-\frac{P_{0}}{4 \mu}$ \\
\hline RVP & $(3),(5),(7)$ & $\left(\frac{1}{R^{3} \omega_{0}}\right) \frac{\partial X(\tau)}{\partial \tau}$ & o & o \\
\hline RAP & $(3),(5),(7)$ & $\left(\frac{1}{R^{3} \omega_{0}^{2}}\right) \frac{\partial^{2} X(\tau)}{\partial \tau^{2}}$ & $-\frac{P_{0}}{4 \mu}$ & $\mathbf{0}$ \\
\hline DIS & $(3),(5),(7),(25)$ & $\frac{u(\mathbf{r}, \tau)}{R}$ & o & $\frac{P_{0}}{4 \mu}\left(\frac{R}{r}\right)^{2}$ \\
\hline VEL & $(3),(5),(7),(25)$ & $\left(\frac{1}{R \omega_{0}}\right) \frac{\partial u(r, \tau)}{\partial \tau}$ & $\frac{\zeta P_{0} R}{2 \mu r}$ & $\mathbf{0}$ \\
\hline ACC & $(3),(5),(7),(25)$ & $\left(\frac{1}{R \omega_{0}^{2}}\right) \frac{\partial^{2} u(r, \tau)}{\partial \tau^{2}}$ & $\infty$ & $\mathbf{0}$ \\
\hline R5s & $(3),(3),(7),(23)$ & $\frac{\sigma_{r}(r, \tau)}{P_{0}}$ & $-\frac{R}{r}$ & $-\left(\frac{R}{r}\right)^{3}$ \\
\hline TSS & (3), (5), (7). (24) & $\frac{\sigma_{t}(\tau, \tau)}{\mathrm{P}_{0}}$ & $-\left(1-25^{2}\right) \frac{R}{r}$ & $\frac{1}{2}\left(\frac{R}{r}\right)^{3}$ \\
\hline MSS & (3), (5), (7), (31) & $\frac{\sigma_{\mu}(\mathrm{r}, \tau)}{\mathrm{P}_{0}}$ & $-\zeta^{2} \frac{R}{r}$ & $-\frac{3}{4}\left(\frac{R}{r}\right)^{3}$ \\
\hline MNS & (3). (3), (7), (33) & $\frac{a_{m}(r, \tau)}{P_{0}}$ & $-\zeta^{2} \frac{\mathrm{kR}}{\mu \mathrm{r}}$ & o \\
\hline RSN & $(3),(5),(7),(41)$ & $\frac{\partial u(r, \tau)}{\partial r}$ & $-\zeta^{2} \frac{\mathrm{P}_{0} \mathrm{R}}{\mu \mathrm{r}}$ & $-\frac{P_{0}}{2 \mu}\left(\frac{R}{r}\right)^{3}$ \\
\hline T8N & (3), (5), (7), (25) & $\frac{u(r, \tau)}{r}$ & o & $\frac{\mathbf{P}_{0}}{4 \mu}\left(\frac{\mathbf{R}}{r}\right)^{3}$ \\
\hline
\end{tabular}

ADP, reduced-dtoplacement potential; RVP, reduced velocity potentiv, RAP, redueed-accelerrion pocentiol, Dis, displecement;
VEL, velocity; ACC, accelerntion! RSS, radiel otrtsi TSS, tangentil ater
MSS, maximum chear atretw, MNS, mean normal otrew; RSN, radil strain, TSN, tengentisl stain. 


\section{CONCLUSIONS}

As shown in Fig. 1, the values of the undamped natural frequency, the damping constant, and the natural frequency for the damped oscillations of a spherical cavity in an elastic solid are in the ratio $a: b:\left(a^{2}-b^{2}\right)^{1 / 2}$.

The undamped natural frequency $\omega_{0}=2 b / R$ and the damping ratio $\zeta=b / a$ are the appropriate parameters for defining the dynamic characteristics of a spherical cavity as a source of compressional waves in an elastic solid. These parameters, $\omega_{0}$ and $\zeta$, are also the appropriate parameters for defining the characteristics of the radial and tangential stresses in the near and far fields. However, R $\omega / a$ is the appropriate parameter for defining the maximum shear and mean normal stresses in the near and far fields, the radial and tangential strains, and the radial displacement, velocity, and acceleration.

The transitions between the near and far fields are functions of both the radial coordinate and the frequency, and are different for the several stresses and strains, and for the radial displacement, velocity, and acceleration.

The solutions for the stresses and strains, and the radial displacement, velocity, and acceleration, are high-frequency far-field solutions for $\tau \rightarrow 0+$ and zero-frequency near-field solutions for $\tau \rightarrow \infty$. 


\section{REFERENCES}

Blake, F. G., Jr., "Spherical Wave Propagation in Solid Media," J. Acoust. Soc, Am. 24, 211 (1952).

Brown, G. S., and A. C. Hall, "Dynamic Behavior and Design of Servomechanisms," Trans. Am. Soc. Mech. Eng. 68, 503 (1946).

Draper, C. S., and G. P. Bentley, "Design Factors Controlling the Dynamic Performance of Instruments,"

Trans. Am. Soc. Mech. Eng. 62, 421 (1940).

Gardner, M. F., ard J. L. Barnes, Transients in Linear Systems (John Wiley \& Sons, Inc., New York, 1942).

Gille, J.-C., M. J. Pelegrin, and P. Decaulne, Feedback Control Systems (McGraw-Hill Book Company, Inc., New York, 1959).

Gurvich, I. I., "The Theory of Spherical Radiation of Seismic Waves," Iz1. Akad. Nauk SSSR, Ser. Fiz. Zemli 10, 45 (1965) [English transl.: Bull. Acad. Sci. USSR, Earth Phys. 10, 684 (1965)].

Herbst, R. F., G. C. Werth, and D. L. Springer, "Use of Large Cavities to Reduce Seismir Waves from: Underground Explosions," J. Geophys. Res. 66, 959 (1961).

Jaeger, J. C., and N. G. W. Cook, Fundamentals of Rock Mechanics (Chapman and Hall, Ltd., London, 1971).

Jeffreys, H., "On the Cause of Oscillatory Movement in Seismograms," Mon. Not. Roy. Astron. Soc., Geophjs. Suppl. 2, 407 (1931).

Kawasumi, H., and R. Yosiyama, "On an Elastic Wave Animated by the Potential Energy of Initial .traint."

Bull. Earthquake Res. Inst., Univ. Tokyo 13, 496 (1935).

Knopoff, L., "Q," Rev. Geophys. 2, 625 (1964).

Meyer, M. L., "On Spherical Near Fields and Far Fields in Elastic and Viscoelastic Solids," J. Mech. Phys. Solids 12, 77 (1964).

Papoulis, A., The Fourier Integral and Its Applications (McGraw-Hill Book Company, Inc., New York, 1962). Rodean, H. C., Nuclear-Explosion Seismology (U.S. Atomic Energy Commission, Oak Ridge, TN 1971).

Selberg, H. L., "Transient Compression Waves from Spherical and Cylindrical Cavities," Ark. Fys. 5, 97 (1952).

Yoshiyama, R., "Note on Earthquake Energy," Bull. Earthquake Res. Inst., Univ. Tokyo 41, 687 (1963). 